\title{
Visual mental rotation of possible and impossible objects
}

\author{
ITIEL E. DROR \\ Miami University, Oxford, Ohio \\ CHANDRA IVEY \\ SUNY Health Science Center, Syracuse, New York \\ and \\ COURTNEY ROGUS \\ Miami University, Oxford, Ohio
}

\begin{abstract}
Participants were tested on two visual mental rotation tasks using three-dimensional "possible" and "impossible" shapes. Both types of stimuli can be easily encoded by their parts and how they are spatially organized. However, while possible shapes can also be easily encoded as a global image, it is more difficult to encode impossible shapes in such a way. Participants visually rotated both types of stimuli at comparable rates, reflecting that local representations were used in the process of visual mental rotation.
\end{abstract}

Visual mental rotation has been of interest to researchers for many years as it encompasses many important issues in cognition. Specifically, it has raised questions about the nature of image representation. Early in the debate, the very existence of nonpropositional representations of images and visual imagery was questioned altogether (Anderson \& Bower, 1973; Pylyshyn, 1973). Although the resolution of this debate has advanced considerably (see Kosslyn, 1994), the exact representational format used in imagery processes has been examined in numerous studies and has remained unresolved. Specifically, in the most highly studied process of imagery, visual mental rotation, researchers are divided as to whether an image is encoded as a global representation (e.g., Cooper \& Podgorny, 1976; Cooper \& Shepard, 1973; Robertson \& Palmer, 1983) or whether the image is encoded by its parts and their spatial organization (e.g., Folk \& Luce, 1987; Pylyshyn, 1979; Yuille \& Steiger, 1982; see Kosslyn, 1981, for more details on the distinction between global and local representational manipulations of mental images). Bethell-Fox and Shepard (1988) suggest that unfamiliar stimuli are rotated via local representations of their parts and that global representations are used for rotating familiar stimuli.

Until now, the question about the format of the representation of images in visual mental rotation has been ad-

This research was supported by an Air Force Research Grant Contract F33615-91-D-0009 award to I.E.D. The authors would like to thank Daniel L. Schacter for providing them with the drawings of the possible and impossible shapes and for comments on an earlier version of this paper. The authors would also like to thank Kimberly Thompson and Donna Stevens, who provided help in proofreading the paper. Correspondence should be addressed to I. E. Dror, Benton Hall, Department of Psychology, Miami University, Oxford, OH 45056 (e-mail: idror@miavx1.acs.muohio.edu). dressed mainly by examining the effects of the complexity of images on the rate of rotation. Specifically, if an image is encoded as a global image, then the complexity of the image should not affect the efficacy of the rotation process; however, if the image is encoded by its parts, then the more complex images should be harder to rotate (see Cooper \& Podgorny, 1976, for more details on the logic of this assertion). However, inconclusive results have emerged from studies that examined whether shape complexity affects the rate of rotation. For example, Cooper and Podgorny (1976) and Robertson and Palmer (1983) have not found a complexity effect, but Folk and Luce (1987) and Yuille and Steiger (1982) have found such an effect. Other studies (e.g., Robertson \& Palmer, 1983 ) have examined the question of global versus local representations in visual mental rotation by using stimuli of large letters composed of smaller letters. The results of their study are inconclusive as they are consistent with both a global and a local representation (see Paquet, 1991 , for details). In the paper presented here, a different approach was used to examine the underlying representational format in visual mental rotation-namely, examining the efficacy of rotating "possible" and "impossible" shapes.

Visual mental rotation is an imagery transformation that requires that an image be mentally rotated in space. It is similar to physical rotation because it requires going through intermediate positions. Thus, rotating greater angular orientations requires more time (Cooper, 1975; Shepard \& Cooper, 1982; Shepard \& Metzler, 1971). The rate of rotation (i.e., the slope of increased response time as a function of angle) reflects the processing capacity of the components involved in visual mental rotation itself; the intercept of the function reflects factors that are not related to the rotation process itself (e.g., encoding the 
stimuli, making the comparison, and producing the response). The use of rotation slopes enables researchers to examine which factors affect the process of visual mental rotation (i.e., cause changes in the slopes of rotation).

In order to address the representational format used in visual mental rotation, drawings that depict threedimensional objects that are structurally impossible were used in two rotation tasks. Whereas possible shapes can be easily encoded as a global representation, encoding impossible shapes as a global representation would make their incongruities explicit. Priming studies show that possible shapes prime better than impossible shapes (Schacter, Cooper, \& Delaney, 1990; Schacter, Cooper, Delaney, Peterson, Tharan, 1991). Priming depends on encoding global representations, which is consistent with the assumption that impossible shapes are not encoded as global representations. Thus, it was assumed that impossible shapes are not encoded as global representations or at least that they cannot be encoded and maintained this way as easily as possible shapes.

In the study reported here, we used stimuli of possible and impossible objects in two visual mental rotation experiments. The differences in encoding the two types of stimuli enabled us to examine the underlying representation of images in mental rotation. If participants used global representations, the structural possibility of an object would affect the rotation rate, and both types of stimuli would be rotated at different rates; whereas, if local representations were used, the structural possibility of the object would not affect the rotation rate, and both types of stimuli would be rotated at the same rate. Hence, it was of interest to compare the slopes of rotation of each type of stimulus in order to discover which underlying representation was used.

\section{EXPERIMENT 1}

In Experiment 1, participants were tested on a visual mental rotation task that used intermixed stimuli of possible and impossible shapes. The participants were required to decide whether two objects were identical or mirror reversed, regardless of their orientation.

\section{Method}

Participants. Sixteen undergraduate students volunteered to participate in the experiment; 8 were male and 8 were female. None of the volunteers had participated in a mental rotation study prior to this experiment.

Materials. We chose 16 shapes ( 8 possible and 8 impossible; see Figure 1 for examples) from those used by Schacter et al. (1990; see also Schacter et al., 1991). Because the task required the participants to determine whether shapes were identical or mirror reversed, only objects that were not symmetrical were used. Mirror reversed images (rather than different images) were used for the "no" trials to force the participants to rotate the entire image. If different images had been used for the "no" trials, the participants might have been able to perform the task on the basis of just one feature - and, hence, would not necessarily have encoded and rotated the entire image. To ensure that both types of stimuli had comparable complexity, we chose shapes that had approximately

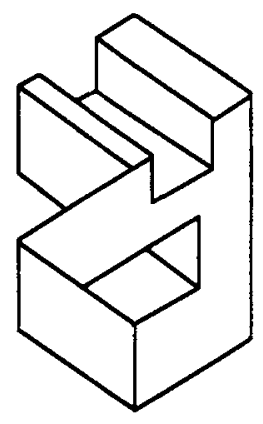

Impossible Shape

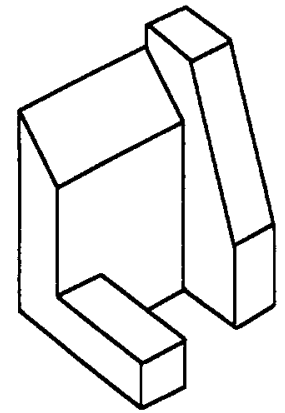

Possible Shape
Figure 1. Example of the stimuli used in the visual mental rotation task.

the same number of lines (see Carrasco \& Seamon, 1996, for a discussion on the importance of complexity of impossible stimuli). All shapes were reduced to a size of approximately $4.4 \times 4.4 \mathrm{~cm}$, corresponding to $5^{\circ} \times 5^{\circ}$ of visual angle from the subject's point of view.

Each one of the 16 shapes was rotated clockwise by $0^{\circ}, 45^{\circ}, 90^{\circ}$, and $135^{\circ}$; for each angular orientation, an identical and mirror-reversed stimulus was made. One hundred and twenty-eight trials were constructed by pairing each of the 16 upright shapes with eight stimuli, which were the identical or mirror-reversed images of the shape at each one of the four orientations. The trials were blocked into eight groups of 16 trials. In each block, each shape appeared once; half the trials presented an identical image, and half presented a mirror-reversed image. Each angular orientation appeared four times. The trials were presented in pseudorandom order, with the constraint that a possible or impossible shape, an angular orientation, or the same response did not appear or occur in more than 3 trials in succession. Two additional shapes (one possible and one impossible) were used to make 16 practice trials. which included all possible combinations of orientations and responses.

Procedure. The participants were tested individually in a single testing session. The participants were seated approximately $50 \mathrm{~cm}$ from the computer screen. The participants first practiced using the keys on the computer keyboard; the "B" key was labeled "yes," and the " $N$ " key was labeled "no." In the 32 keyboard practice trials, the word yes or no appeared on the screen, and the participants were required to press the appropriate key. If a wrong key was pressed, the computer beeped.

After practice with the keys, the participants read the instructions for the task from the computer screen. The set of practice trials was then given. The computer beeped if a wrong response was made, but no such feedback was given during the actual trials. The participants were encouraged to ask questions during the practice, but no talking was allowed during the actual experiment.

The beginning of each trial was indicated by an exclamation point (?) that appeared on the screen for $1,000 \mathrm{msec}$. A shape in the "upright" position was then displayed. The participants studied this shape as long as needed; when they felt that they had memorized it well, they pressed the spacebar. An asterisk $\left({ }^{*}\right)$ then appeared on the screen for $500 \mathrm{msec}$ to indicate that another shape was going to be presented. Following this, the second shape was presented, and the participants were required to decide whether the second shape was an identical or a mirror-reversed image of the first shape, regardless of its orientation in the two-dimensional plane of the computer screen. The participants were instructed to respond as quickly as possible but to remain as accurate as possible.

\section{Results}

Response time (RT) and error rate data were analyzed in an analysis of variance (ANOVA) with angular orien- 
tation and type of stimuli (possible and impossible shapes) as factors. Incorrect responses and outliers were excluded from the RT analysis. An outlier was defined as any score that was larger than 2.5 times the mean of the remaining scores in that cell.

RTs and error rates increased linearly with the degree of angular rotation $[F(3,45)=22.98, p<.01$, for RT, with means of $1,238,1,630,1,744$, and $1,918 \mathrm{msec}$ for $0^{\circ}, 45^{\circ}, 90^{\circ}$, and $135^{\circ}$ of rotation, respectively; $F(3,45)=$ $16.75, p<.01$, for error rate, with means of $9.4 \%, 15.2 \%$, $16.8 \%$, and $25.3 \%$ error for $0^{\circ}, 45^{\circ}, 90^{\circ}$, and $135^{\circ}$ of angular rotation, respectively]. The data reflect the hallmark of visual mental rotation and can be described very well by linear slopes: $\mathrm{RT}=1,309+4.79 \times$ angle, $R^{2}=.93$; error $=9.28+0.11 \times$ angle, $R^{2}=.94$.

The main question was whether or not the type of stimuli (possible and impossible) affected the rate of rotation. As can be seen in Figure 2, there was no interaction be- tween type of stimuli and angular orientation $[F<1$, for $\mathrm{RT} ; F(3,45)=1.93, p=.14$, for error rate].

\section{Discussion}

The participants used visual mental rotation to perform the task, as reflected by linear increases in RTs and error rates as a function of angular rotation. Of particular interest was that the drawings of both the possible and the impossible objects were transformed at comparable rates of rotation. Because impossible objects cannot be easily encoded as global images, the fact that they were rotated at comparable rates to the possible images reflects that stimuli were encoded in local representations that depict images as composed of parts that are spatially organized together.

\section{EXPERIMENT 2}

In Experiment 1, the possible and impossible images were intermixed and counterbalanced. Such a presentation of the stimuli may have induced the participants to
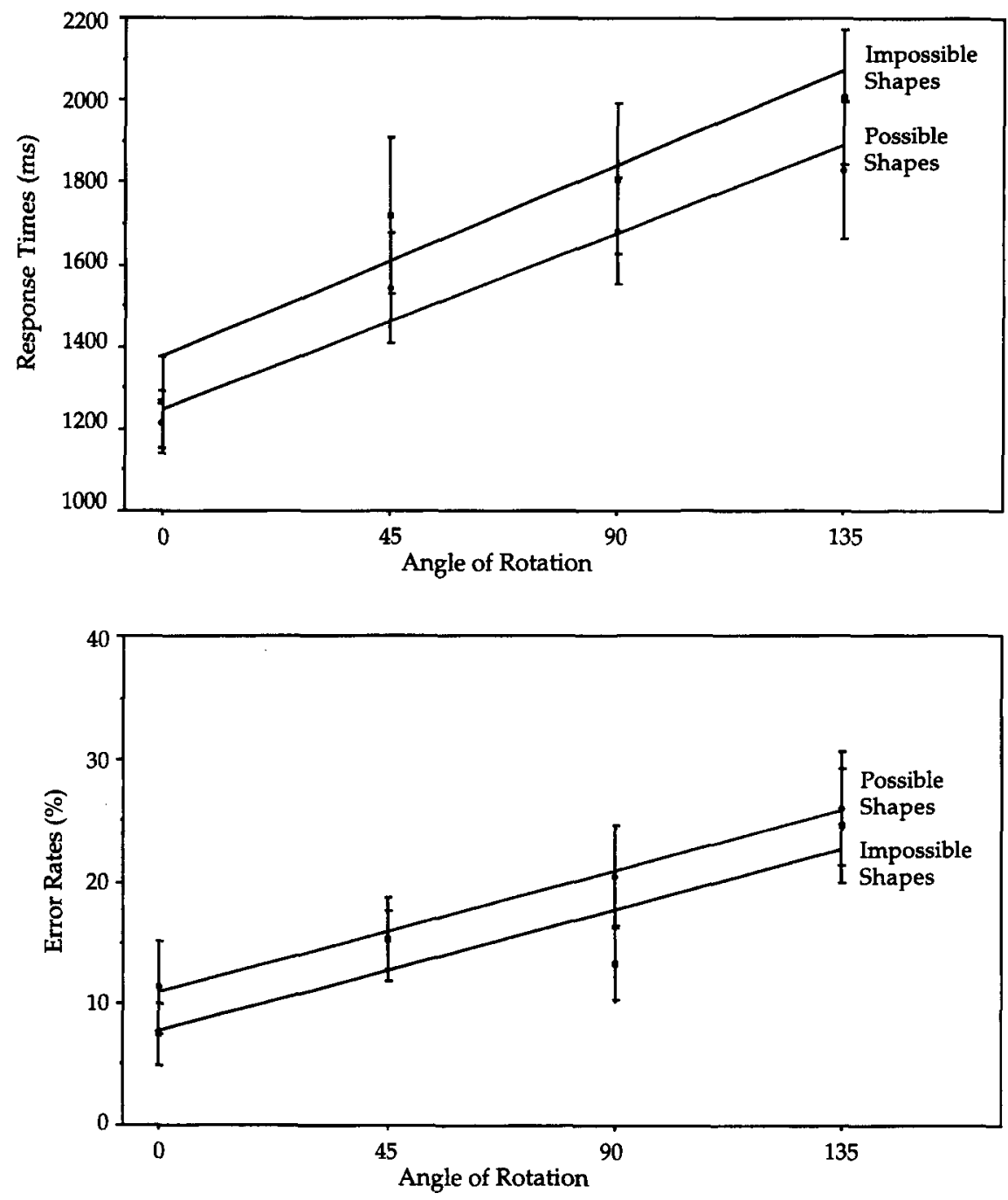

Figure 2. Mean response times (top) and error rates (bottom) as a function of angular rotation. The stimuli of the possible and impossible shapes were intermixed and presented in a single testing session. 
adopt a single rotation strategy that could equivalently deal with both types of stimuli. To address this possibility, we tested participants in two sessions: In one session, we used only stimuli of the possible objects; in the other session, we used only stimuli of the impossible objects.

\section{Method}

Participants. Sixteen undergraduate students volunteered to participate in the experiment; 8 were male and 8 were female. None of the volunteers had participated in a mental rotation study prior to this experiment.

Materials. The same stimuli used in Experiment 1 were used here. The task was divided into two parts. In one part, we included all the stimuli of the possible shapes; in the other part. we included all the stimuli of the impossible shapes. Each part included 64 trials, using eight shapes; each shape appeared twice in four different orientations (once as a "yes" trial, where the shapes were identical; and once as a "no" trial, where the shapes were mirror-reversed images of each other).

Procedure. The same procedure used in Experiment 1 was used here, except that the participants were tested twice, once on one part of the task and once on the other part. The two testing sessions were conducted 2 days apart (on Monday and Wednesday, on Tuesday and Thursday, or on Wednesday and Friday). To counterbalance any effect of practice, half of the participants first performed the part with the possible stimuli and then, in the second session, the part with the impossible stimuli; the other half of the participants performed the task in reverse order.

\section{Results}

The data were analyzed using the same procedures used in Experiment 1. RTs and error rates increased with the degree of angular rotation $[F(3,45)=13.62, p<.01$, for RT, with means of $1,332,1,636,1,666$, and $1,932 \mathrm{msec}$ for $0^{\circ}, 45^{\circ}, 90^{\circ}$, and $135^{\circ}$ of rotation, respectively; $F(3,45)$ $=27.82, p<.01$, for error rate, with means of $7.2 \%$, $16.3 \%, 20.0 \%$, and $29.5 \%$ error for $0^{\circ}, 45^{\circ}, 90^{\circ}$, and $135^{\circ}$ of angular rotation, respectively]. The data reflect the hallmark of visual mental rotation and can be described very well by linear slopes: $R T=1,367+4.07 \times$ angle, $R^{2}=.93$; error $=7.66+0.16 \times$ angle, $R^{2}=.98$.

The main question was whether or not the type of stimuli (possible and impossible) affected the rate of rotation. As can be seen in Figure 3, there was no interaction between type of stimuli and angular orientation $[F<1$, for $\mathrm{RT} ; F(3,45)=1.68, p=.18$, for error rate].

\section{Discussion}

As in Experiment 1, we found that the participants used mental rotation to perform the task and that the rate of rotation was comparable for stimuli of possible and impossible objects. These data were obtained when the participants were tested on only one type of stimuli in a single testing session, suggesting that local representations are preferred in visual mental rotation.

\section{OVERALL ANALYSIS}

The data from each task showed that the participants rotated the stimuli of impossible and possible objects at comparable rates. Although there was no hint of interaction between angular orientation and type of stimuli in either of the tasks, it seemed warranted to further establish the conclusions by analyzing all the data together. Such analysis provides greater statistical power, as reflected in the larger degrees of freedom in the error term. In this analysis, angular orientation and type of stimuli (possible and impossible) were used as within-subjects factors, and pre- sentation (mixed in Experiment 1, and separate in Experiment 2) was used as a between-subjects factor.

Consistent with our earlier analysis, there was no hint of interaction between the type of stimuli and angular orientation $[F<1$. for RT; $F(3,90)=1.56, p=.20$, for error rate]. In fact, the overall analysis with the additional statistical power further reduced the $F$ values of the interactions. Nevertheless, to directly examine the hypothesis that impossible shapes were more difficult to rotate than possible shapes, a linearby-linear contrast was performed. This test revealed no interaction between type of stimuli and angular orientation $\left(F_{\mathrm{S}}<1\right.$, for both RT and error rate).

\section{GENERAL DISCUSSION}

This study set out to examine the nature of image representation used in visual mental rotation - specifically, whether a rotated object is represented as a single global image or as a single image composed of parts that are spatially organized together. To address this issue. the participants were asked to rotate drawings that depicted possible and impossible objects. The drawings of impossible objects used in the present study have been used in a variety of memory tasks (Schacter et al., 1990; Schacter et al., 1991). Those studies established that drawings of impossible shapes are not encoded as global images. The results from the study reported here show that the slopes of rotation for impossible shapes were comparable to those obtained when possible shapes were rotated. The results reflect that image representations used in visual mental rotation are local representations. If shapes were encoded globally, there would have been a change in the rotation rate when the impossible shapes were rotated; however, no such effect was found.

On the basis of the findings obtained in the study reported here, one would predict that the complexity of objects would affect the rate of rotation: If images are encoded locally by their parts and their spatial organization, then more complex images would be harder to rotate (conversely, if images were encoded as a global image, then rotation rates would not be affected by the complexity of the image). Consistent with the prediction derived from the study reported here, other studies have found that complexity did affect the process of visual mental rotation (e.g., Dror \& Kosslyn, 1994: Dror. Kosslyn, \& Waag. 1993: Folk \& Luce, 1987; Yuille \& Steiger, 1982). However. the lack of an effect of the possible/impossible manipulation is not consistent with the conclusions of Cooper and Podgorny (1976). who argued that global representations are used in visual mental rotation. Cooper and Podgorny's argument rests on their observation that the complexity of an image did not affect the process of visual mental rotation.

The inconsistent findings about the effects of complexity, and the findings of other studies that investigated image representation in visual rotation, may result from the different types of stimuli that were used in the various studies and from different task demands. For example, the nature of the stimuli used by Cooper and Podgorny (1976) and their definition of complexity may explain why they did not find a complexity effect. They used random angular shapes and defined complexity by the number of points in a shape. The random shapes that they used are very difficult to encode as local representations: the shapes are not easily described by their constituent parts and how they are spatially organized, as is evidenced by the low verbal association values of the shapes (Vanderplas \& Garvin, 1959). An example of how task demands may play an important role in the results obtained in a study is Pringle and $\mathrm{Cow}$ an's (1978) study. They tested the ability to rotate possible and impossible four-cornered toruses and found that both types of stimuli are rotated in a similar way. However, unlike those in the present study, the shapes in their study were presented simultaneously, which enabled the participants to go back and forth between the two shapes. Thus, the participants did not need to encode the entire image of the retited share (see Kosslyn, 1980, for a more detailed discussion of this issuc). Furthermore, all of Pringle and Cowan's stimuli depieted the same. somewhat symmetrical, four-cornered torus shape. The stimuli differed only in the details of how the torus was constructed. Thus. to perform the task, the participants were encouraged to ignore the global image ant to focus on the details of the draw ing. Tass demands play an importam 

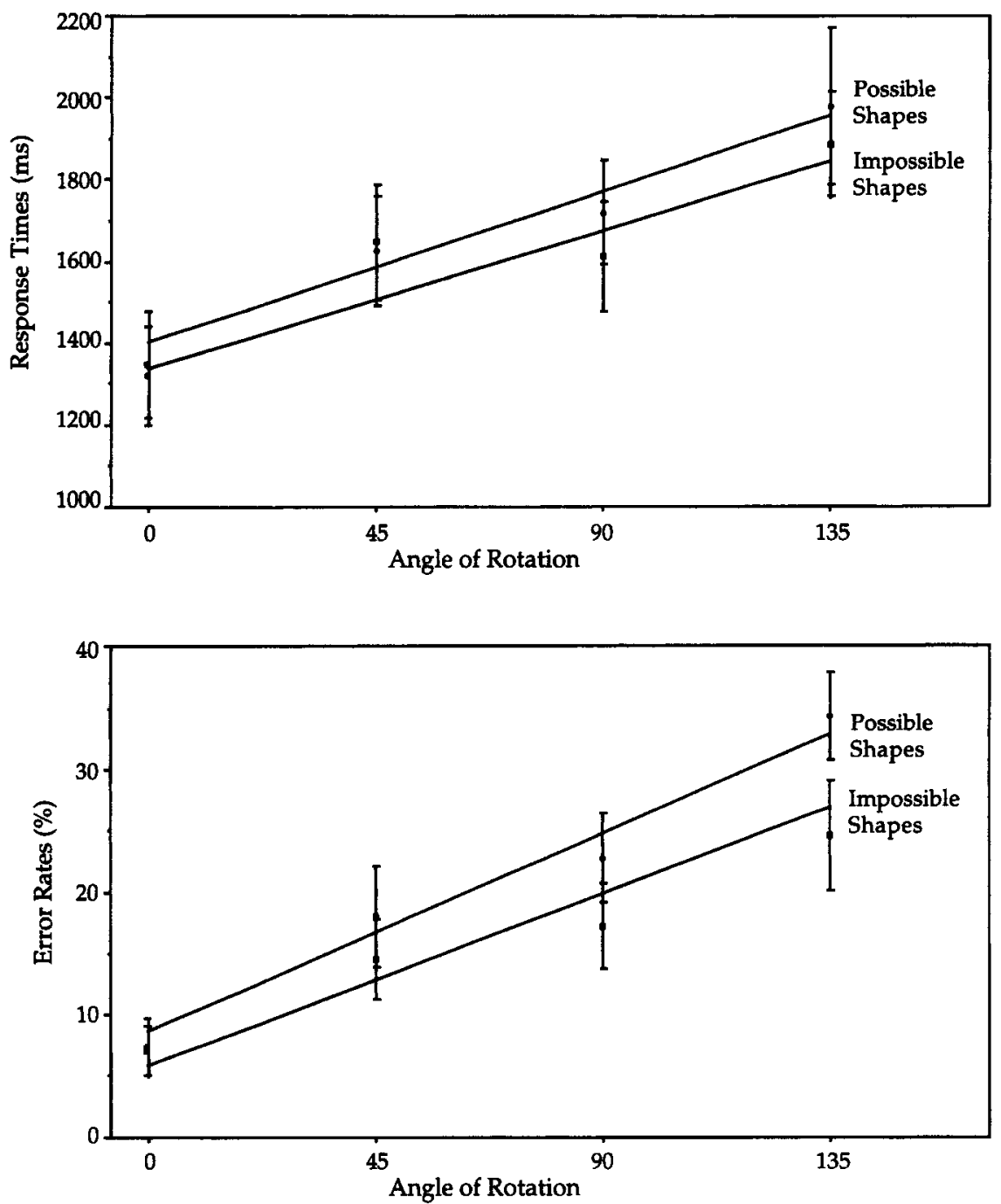

Figure 3. Mean response times (top) and error rates (bottom) as a function of angular rotation. The stimuli of the possible and impossible shapes were separated and presented in different testing sessions.

role in determining whether a global or local representation is used ( $\mathrm{Pa}$ quet, 1991). Given the task demands in Pringle and Cowan's study, it is not surprising that participants did not encode the shapes as global representations.

The study reported here differs from the previous studies that examined the underlying image representation in image rotation. First, most studies examined effects of the complexity of shapes on mental rotation. In this study, we examined the effects of possible and impossible shapes on visual mental rotation. Because impossible shapes are not encoded as global representations (or, at least, they cannot be encoded and maintained this way as easily as possible shapes), the comparison of rotation rates between the two types of stimuli reflects the underlying image representational manipulation. Second, task demands and stimuli induced subjects to prefer one representation over the other. In this study, the stimuli and task demands did not induce a preference for one representation or another. During a trial, stimuli were presented sequentially, which forced the participants to encode the entire shape-they did not have the option to go back and forth between the two shapes. Furthermore, the shapes were all nonsymmetrical, and the "no" trials consisted of mirror-reversed images of the original upright shape; one did not need to focus on the details of the image in order to perform the task, and a single feature did not provide sufficient information to perform the task. Therefore, a global representation of the possible shapes may be easily used to perform the task (either when the stimuli of possible and impossible objects were intermixed or when the stimuli of possible objects were presented by themselves in a single testing session). Despite the fact that global and local representations were viable, participants consistently did not use global representations in visual mental rotation, as was reflected by comparable slopes of rotation for the possible and impossible shapes. This reflects that the mechanisms used in visual mental rotation prefer nonglobal representations.

The results of this study may extend beyond the specific process of visual mental rotation. Local representations may underlie other imagery processes as well. Indeed, studies on the process of image generation have shown that images of letters are generated segment by segment (Kosslyn, Cave, Provost, \& Von Gierke, 1988). Furthermore, the ability to transform images in numerous ways suggests that they are encoded in a way that enables such transformations. For example, the ability to fold a paper in imagery (Shepard \& Feng, 1972) suggests that the overall paper shape is not encoded globally, but rather that the segments and their spatial organization are encoded as separate entities. Such a representation enables easy transformation of visual mental images. Thus, the use of local representations in visual mental rotation is consistent with the goal and nature of the process of image transformation. 


\section{REFERENCES}

Anderson, J. R., \& Bower, G. H. (1973). Human associative memory. New York: V. H. Winston.

Bethell-Fox, C. E., \& Shepard, R. N. (1988). Mental rotation: Effects of stimulus complexity and familiarity. Journal of Experimental Psychology: Human Perception \& Performance, 14, 12-23.

Carrasco, M., \& Seamon, J. G. (1996). Priming impossible figures in the object decision test: The critical importance of perceived stimulus complexity. Psychonomic Bulletin \& Review, 3, 344-351.

COOPER, L. A. (1975). Mental rotation of random two-dimensional shapes. Cognitive Psychology. 7, 20-43.

CoOPER, L. A., \& PoDgorny, P. (1976). Mental transformations and visual comparison processes. Journal of Experimental Psychology. Human Perception \& Performance, 2, 503-514.

CoOper, L. A., \& Shepard, R. N. (1973). Chronometric studies of the rotation of mental images. In W. G. Chase (Ed.), Visual information processing (chap. 3). New York: Academic Press.

Dror, I. E., \& KossLyn, S. M. (1994). Mental imagery and aging. Psychology \& Aging, 9, 90-102.

Dror, I. E., Kosslyn, S. M., \& WAAG, W. (1993), Visual-spatial abilities of pilots. Journal of Applied Psvchologv, 78, 763-773.

FolK, M. D., \& LUCE, D. R. (1987). Effects of stimulus complexity on mental rotation rates of polygons. Journal of Experimental Psychology: Human Perception \& Performance, 13, 395-404.

KosSlyn, S. M. (1980). Image and mind. Cambridge, MA: Harvard University Press.

KossLYN, S. M. (1981). The medium and the message in mental imagery: A theory. Psychological Review, 88, 46-66.

KossLyN, S. M. (1994). Image and brain: The resolution of the imagery debate. Cambridge, MA: MIT Press.

Kosslyn, S. M., Cave, C. B., Provost, D., \& Von Gierke, S. (1988). Sequential processes in image generation. Cognitive Psychology, 20, 319-343.
PAQuer, L. (1991). Mental rotation of compound stimuli: The effects of task demands, practice, and figural goodness. Memory \& Cognition, 19, 558-567.

Pringle, R., \& Cowan, T. M. (1978). Mental rotation of possible and impossible four-cornered toruses. Perception \& Psychophysics. 24, 84-92.

Pylyshyn, Z. W. (1973). The imagery debate: Analogue media versus tacit knowledge. Psychological Review, 87, 16-45.

Pylyshyn, Z. W. (1979). The rate of "mental rotation" of images: A test of a holistic analogue hypothesis. Memory \& Cognition, 7, 19-28.

Robertson, L. C., \& Palmer, S. E. (1983). Holistic processes in perception and transformation of disoriented figures. Journal of Experimental Psychology: Human Perception \& Performance, 9, 203-214.

Schacter, D. L., CoOper, L. A., \& Delaney, S. M. (1990). Implicit memory for unfamiliar objects depends on access to structural descriptions. Journal of Experimental Psychology: General, 119, 5-24.

Schacter, D. L., Cooper, L. A., Delaney, S. M., Peterson, M. A., \& Tharan, M. (1991). Implicit memory for possible and impossible objects: Constraints on the construction of structural descriptions. Journal of Experimental Psychology: Learning, Memory, \& Cognition, 17, 2-19.

ShEPARd, R. N., \& CoOper, L. A. (1982). Mental images and their transformations. Cambridge, MA: MIT Press.

ShePaRd, R. N., \& FENG, C. (1972). A chronometric study of mental paper folding. Cognitive Psychology, 3, 228-243.

ShePARd, R. N., \& METZler, J. (1971, February). Mental rotation of three-dimensional objects. Science, 171, 701-703.

VANDERPlas, J. M., \& Garvin, E. A. (1959). The association value of random shapes. Journal of Experimental Psychology, 57, 147-154.

Yuille, J. C., \& Steiger, J. H. (1982). Nonholistic processing in mental rotation: Some suggestive evidence. Perception \& Psychophysics, 31, 201-209.

(Manuscript received July 12, 1996; revision accepted for publication October $10,1996$. 